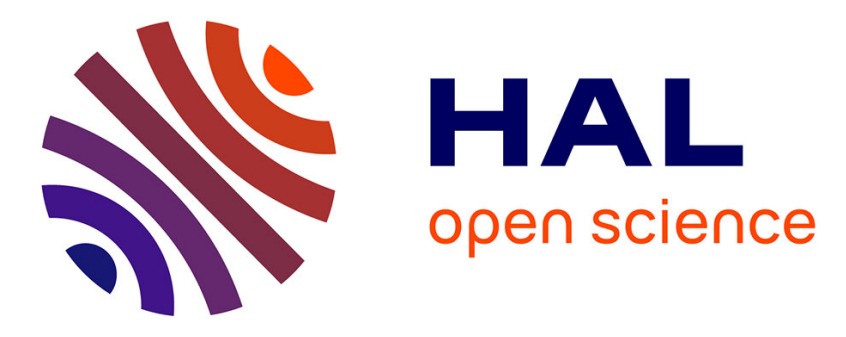

\title{
d-wave superconductivity in the Anderson lattice
}

M. Lavagna, A. J. Millis, P.A. Lee

\section{To cite this version:}

M. Lavagna, A. J. Millis, P.A. Lee. d-wave superconductivity in the Anderson lattice. Journal of Magnetism and Magnetic Materials, 1987, 63-64, pp.432-434. 10.1016/0304-8853(87)90629-9 . hal01896376

\section{HAL Id: hal-01896376 https://hal.science/hal-01896376}

Submitted on 17 Oct 2018

HAL is a multi-disciplinary open access archive for the deposit and dissemination of scientific research documents, whether they are published or not. The documents may come from teaching and research institutions in France or abroad, or from public or private research centers.
L'archive ouverte pluridisciplinaire HAL, est destinée au dépôt et à la diffusion de documents scientifiques de niveau recherche, publiés ou non, émanant des établissements d'enseignement et de recherche français ou étrangers, des laboratoires publics ou privés. 


\title{
d-WAVE SUPERCONDUCTIVITY IN THE ANDERSON LATTICE
}

\author{
M LAVAGNA*+, A J MILLIS $\%+$ and P A Lee ${ }^{+}$ \\ Department of Physics, $M$ I T , Cambridge, MA 02139, USA
}

\begin{abstract}
We show, within a slave-boson $1 / N$ expansion, that the $U=\infty$ lattice Anderson model is unstable to d-wave superconductivity The pairing mechanism is slave-boson exchange between heavy quasiparticles The model is extended to include the Coulomb pseudopotential We discuss the pressure dependence of $T_{\iota}$ in light of our theory
\end{abstract}

The heavy fermion superconductors have been of considerable and contınuing interest to condensed matter physicists since their discovery seven years ago It is now believed that the superconducting state in the heavy fermion materials is "unconventional" in two senses One sense has to do with the form of the gap function, which appears to vanish along points or lines on the fermı surface [1], and implies that heavy fermion superconductivity involves electrons paired in a state of non-zero angular momentum The other, perhaps more fundamental, unconventionality is that heavy fermion superconductivity is unlikely to be due to the conventional electron-phonon interaction [2]

In this paper, we show that the $U=\infty$ Anderson model is unstable to d-wave superconductivity in the same limit in which it describes heavy fermion behavior We use the slave boson large degeneracy $(N)$ formulation, originally devised for the l-impurity [3-5] and recently extended to the lattice [6-8], in which the Hamiltonidn can be written within the constraint $Q_{1}=n_{\mathrm{f}}^{1}+n_{\mathrm{b}}^{1}=1$

$$
\begin{aligned}
H_{\mathrm{F}}= & \sum_{k m} \epsilon_{k} c_{k m}^{+} c_{k m}+E_{0} f_{k m}^{+} f_{k m}+\frac{V}{\sqrt{ } N} \\
& \times \sum_{k q m} c_{k m}^{+} f_{k+q m} b_{q}^{+}+\mathrm{h} \mathrm{c}
\end{aligned}
$$

All energies dre measured from the chemical potential, $\mu$ We are interested in the Kondo limıt,

+ Supported by NSF Grant Number DMR-8521377

* On leave from Laboratorre Lous NÉEL-CNRS, 166x 38042 Grenoble Cédex, France, Supported in part by an NSF-CNRS Exchange Award

Supported by AT\&T Bell Labs, Ph D Scholarshıp in which $-E_{0}>\rho_{0} V^{2} \rho_{0}$ is the c-electron density of states of width $2 W$ To enforce the constraint, one introduces a Lagrange multiplier $\lambda$ The model possesses a $1 / N$ expansion around the mean-field theory The fluctuations may be studied in either the Cartesian or the radial gauge To avoid the complications of infrared divergences appedring in the Cartesian gauge [5], we choose here the radial-gauge $b_{t}^{+}=r_{t} \mathrm{e}^{t_{1} \theta_{1}}$ and $f_{t}=f_{t}^{\prime} \mathrm{e}^{t^{\theta_{1}}}$ The main results of the paper eq (7) could be obtained in the Cartesian gauge, also

We define in this way two bose-fields $r$ and $\lambda(\theta$ being incorporated into $\lambda$ ), and consider their fluctudtions around their mean-field values $r_{0}$ and $\lambda_{0}=\epsilon_{\mathrm{F}}-E_{0}$

$\epsilon_{\mathrm{\Gamma}}=W \mathrm{e}^{-\left|E_{0}\right| / \rho_{0} V^{2}}$,

$V^{2} r_{0}^{2}=\sigma_{0}^{2}=q_{0} \epsilon_{\mathrm{F}} n_{\mathrm{F}} / \rho_{0}$

with

$n_{\mathrm{r}}=\left(1+\epsilon_{\mathrm{F}} / \rho_{0} V^{2}\right)^{-1}$,

$\epsilon_{\mathrm{F}}$ is the Kondo temperature, $q_{0}$ is defined as $Q_{1} / N$, but to obtain a sensible $1 / N$ expansion, we must regard $q_{0}$ ds a parameter independent of the explicit $N$, and only set $q_{0}=1 / N$ at the end of the calculation The mean-field theory describes electrons moving in a bandstructure presentıng a gap around $\epsilon_{F}$ The elementary excitations are heavy quasiparticles of mass enhancement $m^{*} / m=\rho / \rho_{0}=\left(\sigma_{0} / \epsilon_{\mathrm{H}}\right)^{2}=q_{0} / \rho_{0} \epsilon_{\mathrm{F}} \gg 1$

Corrections to the mean-field theory are due to electron-boson interactions of order $\leqslant 1 / N$ In this paper we show that, even though weak, this electron-boson interaction leads to d-wave superconductivity We proceed by investigating 
divergences in the scattering amplitude $\Gamma$ for two quasiparticles of opposite momenta near the fermı surface

The quasiparticle scatterıng amplitude is conventionally decomposed into spin singlet and triplet channels, this is meaningless in our spin $N$ model An alternative procedure is to decompose the scattering amplitude into orbital angular momentum channels Since $\boldsymbol{k}$ and $\boldsymbol{k}^{\prime}$ lie on the fermi surface (see fig 1), $\Gamma$ is a function only of the angle $\varphi$ between them $|\boldsymbol{q}|=\left|\boldsymbol{k}^{\prime}-\boldsymbol{k}\right|=$ $2 k_{\mathrm{F}} \sin \varphi / 2$ The interaction in the 1 th orbital angular momentum channel is

$\Gamma_{l}=(2 l+1) / 2 \int \mathrm{d} \varphi \sin \varphi P_{l}(\cos \varphi) \Gamma(\varphi)$

$\Gamma$ can be expanded in terms of the two-particle irreducible (2PI) vertex, $\Gamma_{0}$ (fig 1) To leadıng order in $1 / N, \Gamma_{0}$ consists of two parts a dressed boson propagator and the matrix elements coupling this to the fermion operators Because $\Gamma_{0}$ is order $1 / N$, we need only consider contributions to $\Gamma$ in which the smallness of $\Gamma_{0}$ is compensated by a logarithmıc divergence arising from a particleparticle propagator $\mathrm{We}$ find that logarithmic divergences only occur when both intermediate lines are in the lower band and the frequency $\omega$ and momentum $p$ are such that $|\omega|,\left|E_{p 1}\right|<\epsilon_{\mathrm{f}}$ In this region we may, to logarithmic accuracy, replace the full interaction by its static limit, $\Gamma_{0}$ $(q, \omega=0)$, and cut off the energy integrals at $\epsilon=\epsilon_{\mathrm{F}}$

After decomposing $\Gamma_{0}$ as in eq (3) we may immediately write

$\Gamma_{l}=\Gamma_{o l} /\left(1+\rho \Gamma_{o l} \ln \epsilon_{\mathrm{F}} / T\right)$

The criterion for $l$-wave superconductivity is $\Gamma_{0 l}<0$

We have computed $\Gamma_{0}(q, \omega=0)$, we find (If

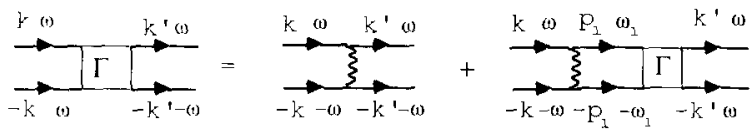

Fig 1 Expansion of $\Gamma$ in terms of $\Gamma_{0}$. The solid lines with arrows are non-interaction Green's functions, $p_{1}$ and $\omega_{1}$ vary over all possible values The wiggly line is the dressed boson propagator discussed in refs $[6,7]$ $\left.\alpha=q_{0} / \mu \rho_{0}\right)$

$\rho \Gamma_{\mathrm{os}}=1 / N-02 \alpha / N, \quad \rho \Gamma_{0 \mathrm{p}}=01 \alpha / N$,

$\rho \Gamma_{\mathrm{Od}}=-007 \alpha / N$

$\Gamma_{o s p}>0$, but $\Gamma_{0 d}<0$, the $\Gamma_{0 l}$ are very small for $l>2$ We therefore expect the boson mediated interaction to give d-wave $(l=2)$ superconductivity

We now extend the Anderson model to include Coulomb interactions involving the $\mathrm{c}$ electrons Two additional terms are possible we write them $U_{\mathrm{cc}} n_{\mathrm{c}}(r) n_{\mathrm{c}}\left(r^{\prime}\right)$ and $U_{\mathrm{fc}} n_{\mathrm{f}}(r) n_{\mathrm{c}}\left(r^{\prime}\right)$ The f-f interaction is already included in the Anderson model We are interested in the short range part of the interaction, $U_{\mathrm{cc}}$ and $U_{\mathrm{fc}}$ are therefore phenomenological fermı liquid parameters expected to be weak $N \rho_{0} U<1$ The $U_{\text {fc }}$ term is negligible [8] A straightforward extension of the usual derivation of the Coulomb pseudopotential [9] leads to a new effective interactions for $l=2$

$\lambda_{\mathrm{eff}}=-\rho \Gamma_{\mathrm{Od}}-\left(m / m^{*}\right) \mu^{*} / N$

Here $\mu^{*}=\mu /\left(1+\mu \operatorname{Ln}\left(W / \sigma_{0}\right)\right)$ is the $l=2$ projection of $N \rho_{0} U_{\text {cc }}$, rescaled via the AndersonMorel scalıng which operates from $W$ to $\sigma_{0}$, where the electrons lose their $c$ character and the scaling breaks down The factor $\left(\mathrm{m} / \mathrm{m}^{*}\right)$ comes from the combination of the small weight of the celectrons at the Fermı surface and the density of states enhancement

Eq (6) 1mmediately yields

$T_{\mathrm{c}}=\epsilon_{\mathrm{f}} \exp \left(-1 / \lambda_{\mathrm{eff}}\right)$

We comment (1) although the attractive part of our interaction is of order $q_{0} / N \sim 1 / N^{2}$, our calculation, which included only $1 / N$ contributions to $\Gamma_{0}$, is consistent Any two boson contributions to $\Gamma_{0}$ would give a term in order $q_{0} / N^{2}=1 / N^{3}$ for $\Gamma_{0 l \neq 0}$ (11) The attractive pairing interaction is specific to the lattice in the 1-1mpurity version of our model, one finds the s-channel interaction is repulsive and all other interaction amplitudes are zero (111) Because the Coulomb term is multiplied by $m / m^{*}$, it is negligible in the Kondo limit, $\eta=-E_{0} / \rho_{0} V^{2} \gg 1$, and superconductivity is possible despite the weakness of the pairng interaction Using eqs (3), (6), (7) one can show that as 
$\eta$ decreases from $\propto, T$, must increase, pass through d maximum at some $\eta=\eta_{\max }$ and then decredse (iv) It seems possible to vary $\eta$ experimentally by applying pressure, $P$ In $\mathrm{Ce}$ and $U$ compounds, $\mathrm{d} \eta / \mathrm{d} P<0$ [1] In $\mathrm{CeCu}_{2} \mathrm{Sl}_{2} T_{\mathrm{c}}(P)$ has the qualitative behavior expected if $\eta(P=$ $0)>\eta_{\max }[10,11]$, In $\mathrm{UPt}_{3}$ [12] and $\mathrm{UBe}_{13}$ [13] $T_{\mathrm{c}}(P)$ monotonically decreases with increasing $P$, as expected if $\eta(P=0)<\eta_{\max }$

In conclusion, we have shown that in the Anderson lattice there is $d$ non-phonon mechanısm which leads to a weak, d-wave superconducting instability, at least withın our $1 / \mathrm{N}$ model The superconductıng instability is specific to the heavy fermion state, and is due to slave boson exchange

\section{References}

[1] For an experımental review see G R Stewart, Rev Mod Phys $56(1984) 755$
[2] P A Lee, T M Rice J W Serene L J Sham and J W Wilkıns, Comments on Solid State Physıcs, vol 12B (1986) p 99

[3] P Coleman, Phys Rev B 29 (1984) 3035

[4] N Read and D M Newns, J Phys C 16 (1983) 3273

[5] N Read, J Phys C 10 (1985) 2651

[6] A J Millis and P A Lee to be published

[7] A Auerbach and K Levm, Phys Rev Lett 57 (1986) 877

[8] M Lavagnd, A J Mills and P A Lee, to be publıshed

[9] Sec, for example, J R Schrieffer, Superconductivitv (W A Benjamın, New York, 1984) chap 6

[10] B Bellarbı, A Benoit, D Jaccard, J M Mignot and H F Braun, Phys Rev B 30 (1984) 1182

[11] L Gao, P H Hor, R L Meng, Z L Du, Z X Zhao and C W Chu Bull Am Phys Soc 31 (1986)

[12] J O Willis, J D Thompson, Z Fisk, A de Visser, J J M Franse and A Menovsky, Phys Rev B 31 (1983) 1654

[13] J A Olsen, R A Fisher, N E Phıllıps, G R Stewart and A L Giorgi, Bull Am Phys Soc 31 (1986)

[14] C J Pethick, D Pines, K F Quader, K S Bedell and G E Brown, Ferm Liquid Theory and the Properties of UPt to be published 